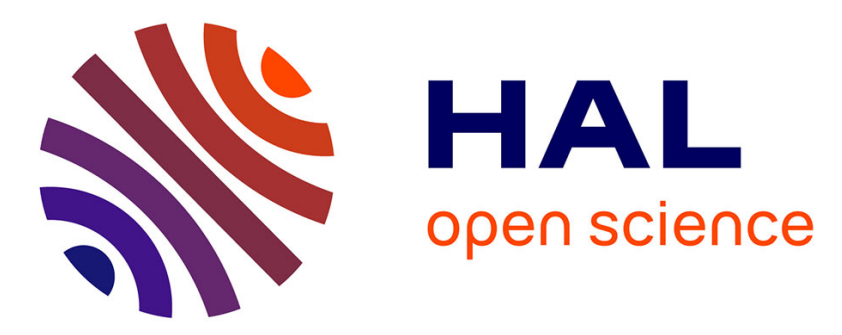

\title{
Etude comparative de quelques cultivars de blé, seigle et triticale dans le Nord du Portugal. I. Productions de grain, de paille, de protéines
} Henrique Guedes-Pinto, Michel Bernard

\section{- To cite this version:}

Henrique Guedes-Pinto, Michel Bernard. Etude comparative de quelques cultivars de blé, seigle et triticale dans le Nord du Portugal. I. Productions de grain, de paille, de protéines. Agronomie, 1983, 3 (7), pp.691-700. hal-00884561

\section{HAL Id: hal-00884561 https://hal.science/hal-00884561}

Submitted on 1 Jan 1983

HAL is a multi-disciplinary open access archive for the deposit and dissemination of scientific research documents, whether they are published or not. The documents may come from teaching and research institutions in France or abroad, or from public or private research centers.
L'archive ouverte pluridisciplinaire HAL, est destinée au dépôt et à la diffusion de documents scientifiques de niveau recherche, publiés ou non, émanant des établissements d'enseignement et de recherche français ou étrangers, des laboratoires publics ou privés. 


\title{
Etude comparative de quelques cultivars de blé, seigle et triticale dans le Nord du Portugal. I. Productions de grain, de paille, de protéines
}

\author{
Henrique GUEDES-PINTO \& Michel BERNARD (*) \\ Instituto Universitario de Tras-os-Montes e Alto Douro, Vila Real, Portugal. \\ * I.N.R.A., Station d'Amélioration des Plantes, F 63039 Clermont-Ferrand.
}

Les résultats d'une expérimentation multilocale réalisée dans la Province de Tràs-os-Montes (Nord-Intérieur) du Portugal, comprenant 17 génotypes de blé et triticale d'origines différentes et 4 populations locales dé seigle, sont présentés. On a tenté, pour cette zone agricole difficile où la production céréalière est faible, de définir les meilleurs cultivars possibles en fonction de leur productivité et de la stabilité de leur rendement. On a étudié la production de grain, de paille, de protéines, les teneurs en protéines et le poids de 1000 grains de ces différents génotypes. Pour tous ces paramètres, les effets lieux et génotypes sont hautement significatifs. Les régressions des productions de chaque génotype à la production moyenne par lieu, ont permis de mettre en évidence 3 groupes principaux de génotypes :

- un groupe dont la production n'est satisfaisante dans aucune condition de culture : il s'agit de 2 blés et de la plupart des triticales CIMMYT de type «Armadillo »;

- un groupe dont la production est satisfaisante en conditions de culture favorables, en sols alluviaux : il s'agit des lignées de triticale I.N.R.A. ;

- un groupe dont la production est satisfaisante en conditions de culture difficiles, sur sols pauvres et climat froid : il s'agit des seigles locaux et du blé local «Barbela ».

Ce dernier groupe présente des productions qui sont d'une remarquable régularité, très faiblement corrélées avec les productions des autres groupes. Un caractère particulier est d'ailleurs la constance de leur poids de 1000 grains.

Ces résultats tracent les axes futurs d'un programme de sélection pour les zones considérées : l'objectif doit être en effet d'obtenir des génotypes garantissant à la fois une production acceptable dans les conditions difficiles et une production élevée dans de bonnes conditions de culture. Ceci implique des plans de croisement utilisant très largement le matériel local, qui doit servir de matériel génétique de base, et du matériel répondant mieux à une élévation du niveau de production potentielle.

Mots clés additionnels : Analyse multilocale, interaction génotype $\times$ milieu, rendement, poids de 1000 grains, teneur en protéines. straw and protein production.

Regional yield trials on 4 cultivars of common wheat, 14 different genotypes of hexaploid triticale (both from CIMMYT and I.N.R.A.) and 4 local cultivars of rye were carried out in 1978 in the Northeastern province of Tras-os-Montes, in Portugal. The trials cover a vast area, including low productivity zones, in which rye is traditionally cultivated. The yield level and its general stability in the area was studied in order to find the cultivars showing the best adaptation to the region. Yield regression for each genotype according to mean local production led us to establish 3 main groups according to the following general traits :

1) unsatisfactory productivity under all conditions : this includes 2 wheat cultivars and most of the CIMMYT triticales of "Armadillo" type ;

2) good productivity under favourable soil conditions; mostly of the alluvial type : the triticales from I.N.R.A. ;

3) fair productivity under difficult environmental conditions, with poor soils and/or cold winters : this group comprises regional rye cultivars and the traditional cultivar "Barbela" of common wheat.

The last group showed remarkable and unique regularity of production in all trials; a very special characteristic of such varieties seems to be constant 1000 grain weight.

Such preliminary results indicate that future breeding programs in this region should be based on obtaining genotypes showing both acceptable productivity under adverse agricultural situations and also responding with high levels of production to favourable environmental conditions. This implies large scale hybridization between well adapted local varieties, as the basic genetic material, and highly productive alien varieties.

Additional key words: Multilocation analysis, genotype - environment interaction, yield, 1000 kernel weight, protein content. 


\section{INTRODUCTION}

La région nord du Portugal est traditionnellement productrice de seigle : près de 75 p. 100 de la surface agricole utile est consacrée à cette céréale, contre 12 p. 100 au blé (ANONYME, 1976). La rusticité du seigle lui permet une meilleure adaptation que les autres céréales aux conditions écophysiologiques, caractérisées par un hiver pluvieux et froid et une fin de printemps et été secs et par des sols de texture sableuse, acides, souvent dépourvus de matière organique (tabl. 1). Ces caractéristiques, jointes à un faible niveau de technicité, expliquent des rendements moyens extrêmement bas (de l'ordre de $10 \mathrm{q} / \mathrm{ha}$ ) $\left({ }^{1}\right)$. Les meilleurs sols des fonds de valléc sont en outre réservés au maraîchage et à la culture de la pomme de terre, à laquelle succède généralement un blé.

Dans ce contexte, il nous a paru intéressant d'expérimenter, dans quelques lieux représentatifs de cette région, un ensemble de cultivars de blés, de seigles régionaux et des lignées de triticale de 2 origines: I.N.R.A. (France) et CIMMYT (Centro Internacional de Mejoramiento de Maiz y Trigo, Mexique).

\section{MATÉRIEL ET MÉTHODES}

\section{A. Le matériel utilisé dans cette expérimentation a consisté en}

- 3 cultivars de blé (Triticum aestivum L. em. Thell) : "Barbela », "Autonomia ", "Siete Cerros".

-4 populations locales de scigle (Secale cereale L.), provenant de Lordelo (Vila Real), Vila Pouca, Montalegre, Gimonde (Bragança).

- 14 lignées de triticale (X. Triticosecale Wittmack) :

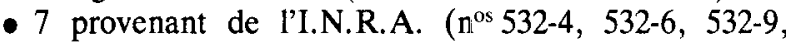
$532-15,532-21,532-43,533-\mathrm{A}-10)$.

- 7 provenant du CIMMYT: "Armadillo", INIAArmadillo S $\left(n^{\circ} 307\right)$, « Maya II - Armadillo S $\left(n^{\circ} 309\right) »$, «Cinnamon $\left(\mathrm{n}^{\circ} 306\right)$ », « Maya I - Armadillo S $\left(\mathrm{n}^{\circ} 310\right)$ », « Maya II - Armadillo $\left(n^{\circ} 308\right)$ » et « Beagle $\left(n^{\circ} 17\right)$ ».

«Barbela» est un blé portugais traditionnellement cultivé dans la province de Tras-os-Montes (région nordintérieure du Portugal) en raison de sa rusticité bien connue. "Autonomia " est une variété d'origine italienne qui a été très cultivée. "Siete Cerros" (obtention du CIMMYT) est un des blés les plus cultivés au Portugal, dans la zone de l'Alentejo principalement (Centre-sud du Portugal), malgré sa sensibilité à la rouille jaune (Puccinia striiformis); il n'est pas bien adapté aux zones Nord du Portugal.

La Brigade technique de la $3^{c}$ Région agricole (Mirandela) a fourni "Armadillo", "Barbela" et "Siete Cerros".

"Autonomia » et les 6 autres lignées de triticale CIMMYT ont été fournies par la Station d'Amélioration des Plantes d'Elvas (Departemento de Plantas de Autofecundaçao).

Comme nous l'avons mentionné, le seigle est la céréale traditionnelle de la région nord. Les populations ont été

${ }^{1}$ ) Cette valeur des statistiques officielles est très probablement imprécise car elle est calculée en fonction de la production totale de grain et de la surface totale semée et ne tient pas compte du fait qu'une partie très importante du semis de seigle est destinée au fourrage. obtenues soit auprès d'agriculteurs, soit auprès du Poste expérimental de Montalegre.

Les lignées de triticale I.N.R.A. expérimentées ont été sélectionnées à la Station d'Amélioration des Plantes de Clermont-Ferrand; elles sont issues de croisements entre Triticale $8 \mathrm{x}$, obtenus par Mme CAUDERON à partir de variétés françaises de blé et de seigle, et $6 \mathrm{x}$, matériel reçu du Dr Jenkins en 1968 (CAUderon \& BERNARD, 1980).

\section{B. Ce matériel a été expérimenté en 1977-78 en 4 localités différentes}

Vila Real ( 2 essais : TT 2 et TT 3), Carlão (C), Montalegre $(\mathrm{M})$ et Bragança $(\mathrm{B})$, correspondant à une gamme de conditions de fertilité de sol et de climat de la région.

Les 2 implantations de Vila Real sont pédologiquement différentes: l'une (TT 2) est sur sol de vallée, de texture franche, humide, trés fertile ; l'autre (TT 3) est sur sol de colline plus pauvre. L'essai de Carlão est sur sol un peu semblable au TT 2 de Vila Real. A Montalegre, on pratique une rotation pomme de terre/seigle/pois ; malgré une certaine fertilité du sol, c'est l'altitude $(1005 \mathrm{~m})$ qui est le facteur limitant et qui impose la culture du seigle dans cette vaste zone de plateau. Enfin, l'implantation à Bragança est sur sol pauvre où l'on pratique habituellement une rotation du type pois/seigle. Tous ces sols sont arides et de texture généralement franco-sableuse (tabl. 1).

La figure 1 donne une idée des différents climats (moyennes annuelles sur 30 ans). Vila Real et Carlão sont les situations les plus chaudes (moyennes annuelles : $13,6{ }^{\circ} \mathrm{C}$; moyenne des minima : $-7,2$ en janvier), Montalegre la plus froide : (moyenne annuelle $9,5^{\circ} \mathrm{C}$ et moyenne des minima $-11,6^{\circ} \mathrm{C}$ en février). Quant à la pluviosité, elle varie de $1450 \mathrm{~mm}$ (Montalegre) à $650 \mathrm{~mm}$ (Carlão).

Pour chaque essai, le dispositif adopté a été celui des blocs randomisés à 3 répétitions, avec une surface parcellaire de $5 \mathrm{~m}^{2}$, à l'exception de TT 3 où la superficie disponible a permis d'installer un essai à 6 répétitions avec une surface parcellaire de $10 \mathrm{~m}^{2}$. La densité de semis a été uniformément de $120 \mathrm{~kg} / \mathrm{ha}$. La fumure a été appliquée en 2 apports: une fumure de fond NPK de 40-80-80, suivie d'une fumure de couverture de $60 \mathrm{uN}$ à la montaison. Ce schéma correspond à celui conseillé par les techniciens agricoles de la région.

Pour chaque parcelle, ont été mesurés les poids de grain (PG) et de paille (PA). Sur un échantillon prélevé dans la récolte, mélange des différentes parcelles de la même variété, ont été mesurés les poids de 1000 grains (PMG) et les teneurs en protéines (TP), évaluées par le laboratoire de Technologie de l'EPAC (Empresa Publica de Abastecimento de Cereais) ce qui a permis d'évaluer une production de protéines (PP) par ha et par génotype.

Un certain nombre d'autres paramètres de production ont été analysés dans une publication précédente (GUEDES Pinto \& Bernard M., 1980).

\section{RÉSULTATS}

\section{A. Production de matière sèche}

Une analyse statistique portant sur les productions de grain et de paille dans les 5 lieux par les 21 cultivars étudiés met en évidence des effets « milieux », "variétés » et "interactions" hautement significatifs, avec un F «milieux 》 généralement supérieur au $F$ «variétés » (tabl. 2). 
TABLEAU 1

Caractères pédologiques des implantations.

Pedological characteristics of yield trial locations.

\begin{tabular}{cccccccc}
\hline Sol & $\begin{array}{c}\text { Altitude } \\
\mathrm{m}\end{array}$ & $\mathrm{pH}\left(\mathrm{H}_{2} \mathrm{O}\right) \mathrm{pH}(\mathrm{KCl})$ & $\begin{array}{c}\text { Mat. org. } \\
\%\end{array}$ & $\begin{array}{c}\mathrm{p} \\
\text { extractible }\end{array}$ & $\begin{array}{c}\mathrm{K} \\
\text { extractible }\end{array}$ & Texture \\
\hline $\begin{array}{c}\text { Villa Real } \\
\text { TT2 }\end{array}$ & 420 & 5,5 & 4,2 & 2,7 & 35 & 70 & franche \\
\hline $\begin{array}{c}\text { Vila Real } \\
\text { TT3 }\end{array}$ & 467 & 5,1 & 3,8 & 1,2 & 15 & 160 & franco-sableuse \\
\hline $\begin{array}{c}\text { Carlão } \\
\text { TTC }\end{array}$ & 610 & 5,8 & 4,3 & 3,8 & 425 & 250 & franco-sableuse \\
\hline $\begin{array}{c}\text { Montalegrc } \\
\text { TTM }\end{array}$ & 1005 & 5,5 & 4,2 & 6,4 & 70 & 115 & franco-sableuse \\
\hline $\begin{array}{c}\text { Bragança (Gimonde) } \\
\text { TTB }\end{array}$ & 691 & 5,7 & 3,9 & 0,7 & 15 & 140 & franco-sableuse \\
\hline
\end{tabular}
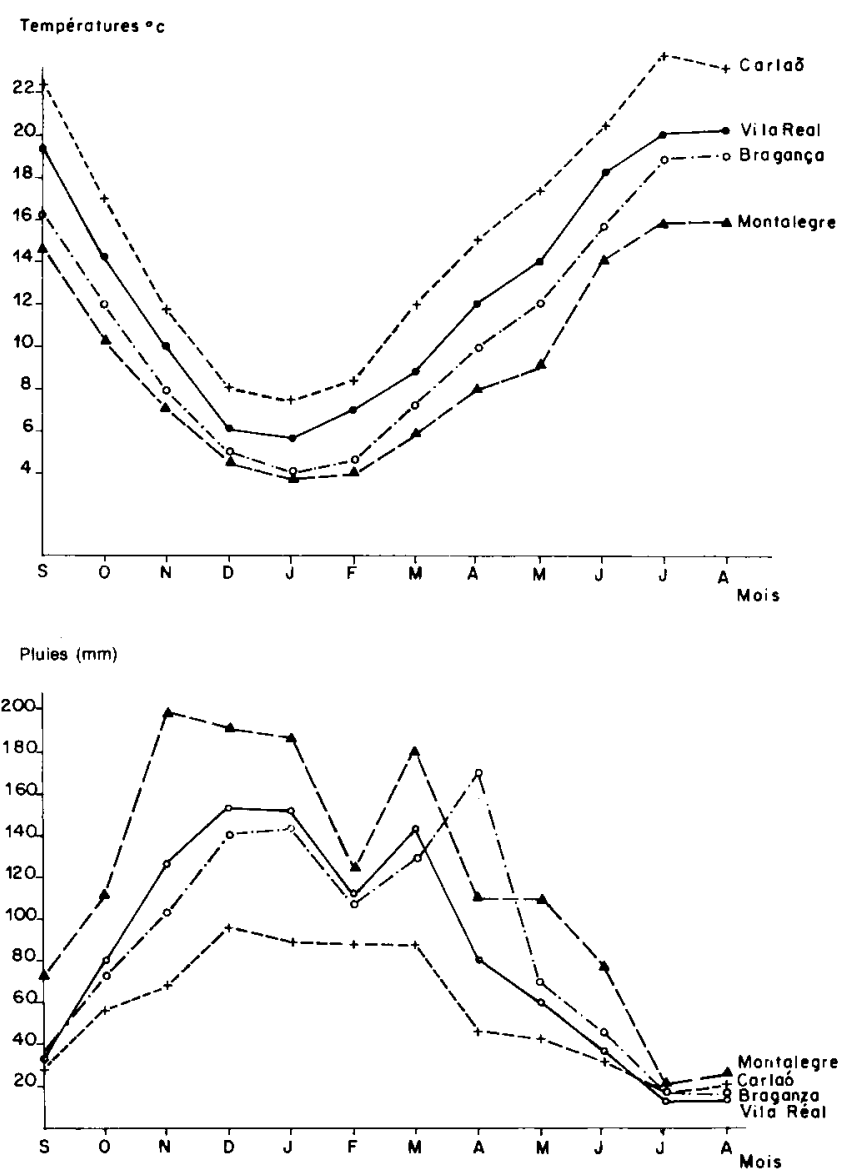

Figure 1

Caractéristiques climatiques des différents lieux d'expérimentation.

a) Températures moyennes mensuelles (établies sur 30 ans).

b) Précipitations moyennes mensuelles (établies sur 30 ans).

Climatic characteristics of yield trial location.

a) Monthly mean temperature (over 30 years).

b) Monthly mean rainfall (over 30 years).
De fait, il est clair que les productions moyennes de grain et de paille sont très différentes dans les différents milieux (tabl. 3) : de 10 à 35 q/ha pour la production de grain, de 28 à 134 pour la production de paille. Le classement par ordre de production décroissante est le suivant : Vila Real TT 2, Carlāo, Montalegre, Vila Real TT 3, enfin Bragança. Ce classement s'explique à la fois par les données climatiques (Montalegre et Brangaça étant des milieux froids) et agronomiques (Vila Real TT 2 et Carlão sont les milieux les plus riches).

La gamme des productions obtenues allant de 1 à 4 permet de penser que l'échantillonnage de nos 5 milieux est représentatif de l'ensemble des milieux agroclimatiques de la province. Notre propos sera de définir quels sont les "génotypes" les mieux adaptés à ces différents milieux (dans la suite, nous utiliserons souvent le terme "génotype » pour désigner un cultivar, une lignée, ou une population locale).

Une $1^{\text {re }}$ approche consiste à examiner les performances moyennes obtenues par les représentants des différents groupes (tabl. 4). En tête, on trouve les triticales I.N.R.A. avec des productions moyennes de grain variant de 27 à $40 \mathrm{q} /$ ha et des productions de paille allant de 72 à $85 \mathrm{q} / \mathrm{ha}$. Puis viennent les seigles ( 24 à $28 \mathrm{q} /$ ha de grain, 70 à $75 \mathrm{q}$ de paille). Le groupe des blés, à l'évidence hétérogène, fournit des productions assez comparables à celles des triticales du CIMMYT : de 9 à $20 \mathrm{q} /$ ha de grain pour le $1^{\text {er }}$ groupe, de 6 à 17 pour le $2^{\mathrm{c}}$, de 38 à $66 \mathrm{q} /$ ha de paille pour les blés, de 48 à 59 pour les triticales CIMMYT. On remarque que si les productions moyennes de grain varient dans un rapport de 1 à 6 , les productions de paille ne varient que dans un rapport de 1 à 2,2 pour l'ensemble des génotypes ; ceci concrétise le fait, perceptible à la lecture du tableau 2 , que le rôle du génotype est plus important au niveau des productions de grain qu'au niveau des productions de paille.

Si l'on tente de percevoir simplement les variations de la production de grain pour chacun des génotypes en fonction des productions moyennes observées dans les différents 
TABLEAU 2

$$
\begin{gathered}
\text { Analyse de la variance - Dispositif multilocal. } \\
- \text { Production de grain. } \\
- \text { Production de paille. } \\
\begin{array}{c}
\text { Analysis of variance - Multilocation yield trial. } \\
- \text { Grain production. } \\
- \text { Straw production. }
\end{array}
\end{gathered}
$$

\begin{tabular}{|c|c|c|c|c|c|}
\hline $\begin{array}{l}\text { Origine } \\
\text { de la variation }\end{array}$ & SCE & DDL & $\mathrm{CM}$ & $F$ & $\begin{array}{c}\text { F } 0,05 \\
\text { table }\end{array}$ \\
\hline Totalc & 99317 & 377 & 263,4 & & \\
\hline Blocs & 804,6 & 13 & 61,9 & $3,17^{(1)}$ & 1,76 \\
\hline Varićtés & 42902,6 & 20 & 2145,1 & 110,05 & 1,61 \\
\hline Lieux & 28767,1 & 4 & 7191,8 & 368,94 & 2,40 \\
\hline Interaction & 21775,6 & 80 & 272,2 & 13,96 & 1,32 \\
\hline Résiduelle & 5068,1 & 260 & 19,49 & & \\
\hline
\end{tabular}

\begin{tabular}{|c|c|c|c|c|c|}
\hline $\begin{array}{l}\text { Origine } \\
\text { de la variation }\end{array}$ & SCE & DDL & $\mathrm{CM}$ & $\mathrm{F}$ & $\begin{array}{c}\text { F } 0,05 \\
\text { table }\end{array}$ \\
\hline Totale & 672555,1 & 377 & 1783,96 & & \\
\hline Blocs & 5546,1 & 13 & 426,63 & $2,82^{(1)}$ & 1,76 \\
\hline Variétés & 83432,0 & 20 & 4171,60 & 27,59 & 1,61 \\
\hline Lieux & 456356,0 & 4 & 114089,0 & 754,66 & 2,40 \\
\hline Interaction & 87914,1 & 80 & 1098,93 & 7,27 & 1,32 \\
\hline Résiduelle & 39306,9 & 260 & 151,18 & & \\
\hline
\end{tabular}

Production de grain

(1) Significatif dans l'essai de CARLÀO.

Production de paillc

\begin{tabular}{|c|c|c|c|c|c|c|}
\hline Production & Lieux & TT2 & $\begin{array}{l}\text { Vila Real } \\
\text { TT3 }\end{array}$ & Carlão & Montalegre & Bragança \\
\hline Grain & & 34,5 & 14,3 & 29 & 17,2 & 10,4 \\
\hline Paille & & 134,4 & 45,4 & 72,3 & 48,1 & 28,1 \\
\hline
\end{tabular}

(1) Significatif dans l'essai de Vila Real TT3.

TABLEAU 3

Productions moyennes de grain et de paille dans les différents milieux ( $q / h a)$. Average of grain production and straw production in the different locations.

milieux (WRIGHT, 1976), par le calcul d'une équation de régression (tabl. 4), il apparaît 3 groupes de génotypes :

1) un groupe présentant des productions moyennes assez faibles (inférieures à $20 \mathrm{q}$ ), avec des pentes d'équations de régression sensiblement inférieures à l'unité, des ordonnées à l'origine peu différentes de zéro : ce groupe comprend les triticales CIMMYT et 2 blés ("Autonomia" et "Siete Cerros »). Les productions des génotypes de ce groupe sont toujours peu importantes, quelles que soient les productions espérées dans les différents milieux;

2) un groupe présentant des productions moyennes assez élevées (supérieures à 20 q), avec des pentes d'équations de régression inféricures à l'unité, des ordonnées à l'origine relativement élevées (12 à $18 \mathrm{q} / \mathrm{ha})$ : ce groupe correspond aux populations locales de seigle et inclut le blé «Barbela » dont le comportement global n'est pas très différent de celui des seigles ;
3) enfin, un groupe présentant des productions moyennes élevées (de l'ordre de $20 \mathrm{q}$ ou plus), avec des coefficients de régression de l'ordre de 2 et des ordonnées à l'origine négative; ce groupe correspond aux triticales I.N.R.A.

Les comportements « moyens » de ces différents groupes sont schématisés sur la figure 2 . Il est évident que les génotypes du groupe 1 ne sont pas d'utilisation intéressante pour la zone d'expérimentation. Par contre, les génotypes appartenant aux groupes 2 (« variétés locales») et 3 (triticales I.N.R.A.) peuvent assurer des productions convenables, les uns en milieu «pauvre de montagne », les autres en milieu « riche de vallée ». De fait, les triticales I.N.R.A. occupent les premières places dans les milieux relativement fertiles et chauds (TT 2, TTC, TT 3) et ont tendance à s'effacer devant les seigles dans les milieux froids ou peu fertiles (Montalegre, Bragança). 


\section{TABLEAU 4}

Production de grain et de paille fournie par les différents génotypes $(y)$, équation des régressions $y_{i j}=b y . j+c$ à la production moyenne de chaque lieu $(y . j)$, coefficients de corrélation $r$ de ces régressions.

Grain and straw production obtained from the different genotypes: means $\left(y_{i}\right)$, regression equations $y_{i j}=b y . j+c$ on the mean production by location $(y, j)$, correlation coefficients $r$ for these regressions.

\begin{tabular}{|c|c|c|c|c|c|c|c|c|c|}
\hline & \multicolumn{4}{|c|}{ Grain } & \multicolumn{4}{|c|}{ Paille } & \multirow{2}{*}{$\begin{array}{c}\begin{array}{c}\text { Harvest } \\
\text { Index }\end{array} \\
\text { moyen }\end{array}$} \\
\hline & yi . & $b$ & c & $r$ & yi. & b & $\mathrm{c}$ & $r$ & \\
\hline Blés Barbela & 20,5 & 0,40 & +12 & $0,65 *$ & 65,6 & 1,12 & $-8,1$ & $0,99 * *$ & 0,31 \\
\hline Autonomia & 9,0 & 0,53 & $-2,1$ & 0,59 & 38,4 & 0,86 & $-18,1$ & $0,96 * *$ & 0,23 \\
\hline Sicte Cerros & 10,3 & 0,90 & $-8,7$ & $0,95 * *$ & 40,7 & 1,16 & $-35,4$ & $0,97 * *$ & 0,25 \\
\hline Seigles Vila Pouca & 28,0 & 0,56 & $+16,3$ & 0,48 & 70,3 & 0,47 & $+39,4$ & 0,72 & 0,40 \\
\hline Lordelo & 22,4 & 0,20 & $+18,2$ & 0,43 & 73,6 & 0,42 & $+45,7$ & 0,62 & 0,32 \\
\hline Montalegre & 24,2 & 0,29 & $+18,0$ & 0,48 & 70,0 & 0,53 & +35 & $0,85^{*}$ & 0,35 \\
\hline Gimonde & 24,2 & 0,33 & $+17,3$ & 0,59 & 74,8 & 0,56 & $+38,2$ & 0,76 & 0,32 \\
\hline \multicolumn{10}{|l|}{ Triticale INRA } \\
\hline $532-43$ & 33,7 & 1,96 & $-7,6$ & $0,96^{* *}$ & 81,7 & 0,65 & $+20,6$ & $0,97^{* *}$ & 0,41 \\
\hline $533-A-10$ & 26,6 & 1,64 & $-7,9$ & $0,98^{* *}$ & 72,2 & 1,26 & $-10,3$ & $0,99 * *$ & 0,37 \\
\hline $532-6$ & 30,9 & 1,76 & $-6,2$ & $0,97 * *$ & 82,7 & 1,39 & $-8,4$ & $0,99 * *$ & 0,37 \\
\hline 21 & 35,5 & 2,10 & $-8,8$ & $0,97^{* *}$ & 82,0 & 1,33 & $-5,7$ & $0,99 * *$ & 0,43 \\
\hline 15 & 36,2 & 2,20 & $-10,3$ & $0,98^{* *}$ & 85,0 & 1,45 & $-10,5$ & $0,98 * *$ & 0,43 \\
\hline 9 & 32,0 & 1,95 & $-9,0$ & $0,96^{* *}$ & 82,8 & 1,38 & $-8,1$ & $0,98 * *$ & 0,30 \\
\hline 4 & 40,0 & 2,29 & $-8,35$ & $0,97 * *$ & 83,9 & 1,49 & $-14,1$ & $0,99 * *$ & 0,48 \\
\hline \multicolumn{10}{|l|}{ Triticale CIMMYT } \\
\hline Armadillo & 6,6 & 0,22 & $+1,9$ & 0,74 & 53,5 & 0,66 & $+10,6$ & $0,96 * *$ & 0,12 \\
\hline Inia-ArmS (307) & 7,0 & 0,23 & $+2,2$ & 0,66 & 53,7 & 0,87 & $-3,5$ & $0,98^{* *}$ & 0,12 \\
\hline Maia II-ArmS (309) & 8,6 & 0,39 & $+0,4$ & $0,84 *$ & 48,2 & 0,73 & $+0,2$ & $0,98 * *$ & 0,18 \\
\hline Cinnamon (306) & 5,8 & 0,21 & $+1,5$ & $0,81 *$ & 54,3 & 0,81 & $+1,1$ & $0,99 * *$ & 0,11 \\
\hline Maia I-ArmS (310) & 8,0 & 0,47 & $-1,9$ & $0,92 * *$ & 51,8 & 0,86 & $-4,6$ & $0,96 * *$ & 0,15 \\
\hline Maia II-ArmS (308) & 15,4 & 1,05 & $-9,2$ & $0,86^{*}$ & 54,5 & 0,96 & $-8,5$ & $0,98 * *$ & 0,28 \\
\hline Beagle (17) & 17,4 & 0,97 & $-3,1$ & $0,85^{*}$ & 59,2 & 1,13 & $-14,9$ & $0,99 * *$ & 0,29 \\
\hline
\end{tabular}

(1) Défini comme le rapport $\frac{\text { grain }}{\text { paille }}$

( $)$ yij = production espérée du génotype $\mathrm{i}$ dans le milieu $\mathrm{j}$.

$\mathrm{y}, \mathrm{j}=$ production moyenne observée dans le milieu $\mathrm{j}$.

(1) Defined as : grain/straw ratio.

(2) $y_{i j}=$ expected production of genotype $i$ in environment $j$.

$y \cdot j=$ average production observed in environment $j$.

On retrouve également les mêmes phénomènes pour ce qui concerne la production de paille, mais avec une ampleur moindre (coefficients de régression davantage regroupés autour de l'unité).

\section{B. Analyse des autres caractères étudiés}

Comme il a été dit plus haut, les poids de 1000 grains et les teneurs en protéines ont été mesurés sur les mélanges des différentes parcelles correspondant à un même génotype dans un même milieu. Pour l'analyse de variance, nous avons donc fait appel au modèle établi par MANDEL (1971), ćtudié en France par LEFORT (1977) \& BRIAN (1978) pour les applications agronomiques, qui permet de travailler sur des tables à 2 voies sans répétition. Nous avons procédé de même pour les productions de protéines par ha des différents génotypes, évaluées comme le produit de la production de grain par la teneur en protćines.
Ce modèle permet de dégager des effets lignes (génotypes) et colonnes (lieux), de tester l'interaction et de la structurer en une somme de termes multiplicatifs. Le modèle complet s'écrit (BRIAN, 1978):

$$
\mathrm{y}_{\mathrm{ij}}=\mathrm{u}+\mathrm{Ll}_{\mathrm{j}}+\mathrm{Cc}_{\mathrm{j}}+\theta_{1} \mathrm{u}_{\mathrm{ii}} \mathrm{v}_{\mathrm{lj}}+\theta_{2} \mathrm{u}_{2 \mathrm{j}} \mathrm{v}_{2 \mathrm{j}}+---+\mathrm{e}_{\mathrm{ij}} \text {. }
$$

Pour les 3 caractères étudiés suivant ce modèle, les résultats de l'analyse de variance sont présentés dans le tableau 5, tandis que les valeurs moyennes obtenues par chacun des génotypes sont présentées dans le tableau 6, dans chacun des lieux par le tableau 7.

\section{Poids de 1000 grains}

A l'intérieur de chacune des 4 familles de génotypes, le rendement est corrélé positivement avec le poids de 1000 grains (de $r=+0,45$ pour les seigles $(S)$ à $r=+0,82$ pour les triticales CIMMYT (H.S.) (fig. 3). Si l'on établit pour le paramètre poids de 1000 grains, les régressions et 


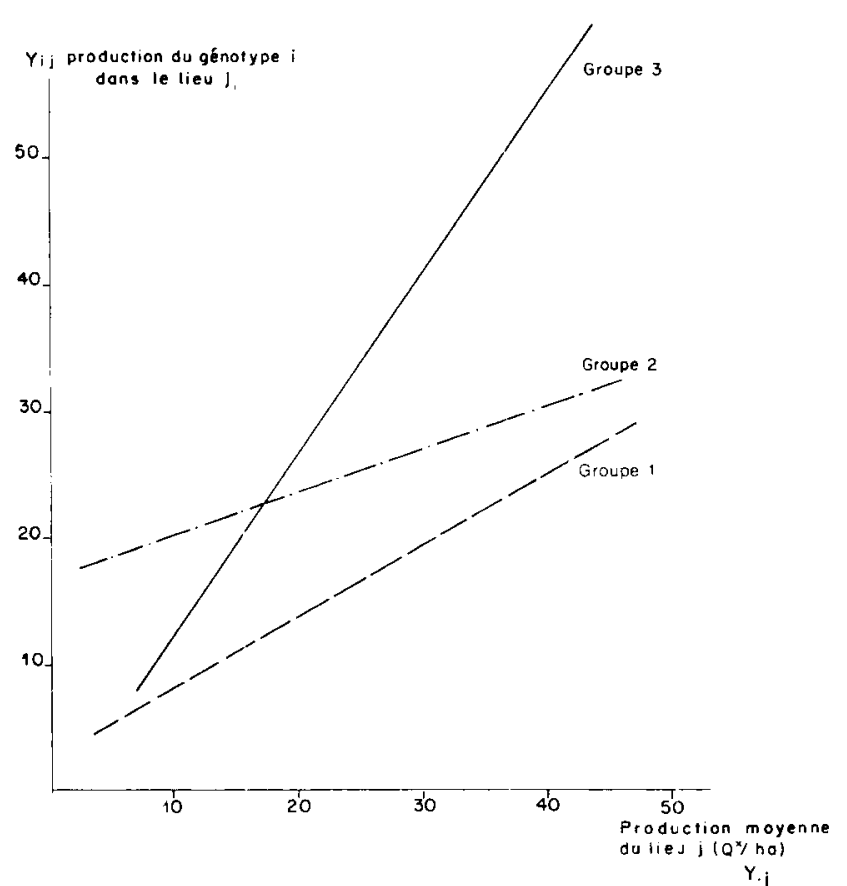

Figure 2

Schématisation du comportement des différents groupes de génotypes par les droites de régression de leur production $y_{i j}$ à la production moyenne observée $y . j$ dans le milieu $j$.

Ordonnée : production du génotype i dans le lieu $j$.

Schematic behaviour of the different groups of genotypes by the regression of their individual production $y_{i j}$ on the mean local production $y . j$.

corrélations $y_{\mathrm{ij}} / \mathrm{y}, \mathrm{j}$, on s'aperçoit que les triticales et les 2 variétés de blé "Autonomia » et "Siete Cenos" varient dans le même sens que les moyennes y . j pour chaque lieu, avec des coefficients de corrélation compris entre 0,91 et 0,99 (H.S.) et des coefficients de régression égaux ou supérieurs à 1. Par contre, le groupe des seigles et la variété de blé «Barbela» présentent, pour ce caractère, des coefficients de régression et de corrélation très voisins de 0 par rapport aux moyennes des licux y.$j$. Cela peut significr que, dans le cas particulier de ces cultivars, le rendement s'établit de façon relativement indépendante du poids de 1000 grains, qui par ailleurs fluctue très peu en fonction des conditions de milieu. Il s'agit évidemment d'une particularité physiologique très intéressante.

\section{Production de protéines}

Aussi bien pour ce qui concerne les teneurs du grain en protéines que pour les quantités de protéines produites à l'hectare, les effets "lieu " et "variété " sont hautement significatifs (tabl. 5).

Les teneurs sont relativement élevées dans les milieux où la production de matière sèche est elle-même élevée: presque 13 p. 100 de moyenne à Vila Real TT 2 , moins de 10 p. 100 en TT 3, à Montalegre et Bragança (tabl. 7). Pour ce qui concerne les valeurs moyennes par génotype (tabl. 6), elles sont généralement faibles (moins de 10 p. 100), sauf pour les triticales CIMMYT (de 12,4 à $15,5$ p. 100$)$.

La classique liaison négative : production de grain/ha teneur en protéines (JESTIN, 1974 ; BERNARD et al., 1979) n'apparaît pas si l'on considère globablement l'ensemble des génotypes et l'ensemble des lieux. Au contraire, elle apparaît très nettement si l'on considère chaque lieu séparément (fig. 4), avec des coefficients de corrélation hautement significatifs (de l'ordre de $-0,7$ à $-0,9$ ). Il s'ensuit qu'à unc production en grain donnée correspond une teneur en protéines d'autant plus élevée que la production moyenne du lieu considéré est plus élevée.

La production de protéines (exprimée en $\mathrm{kg} / \mathrm{ha}$ ) est donc fortement dépendante du milieu : elle varie de $380 \mathrm{~kg} / \mathrm{ha}$ (TT 2) à $95 \mathrm{~kg} /$ ha (Bragança). L'étendue génotypique est un peu plus faible (de $330 \mathrm{~kg}$ à $90 \mathrm{~kg}$ protéines/ha). Si l'on raisonne sur les moyennes génotypiques, on voit que la production de protéines est corrélée positivement avec la production de grain ( $\mathrm{r}=+0,82)$ (fig. 5), négativement avec la teneur en protéines $(\mathrm{r}=-0,77$ ) (fig. 6 ), ce qui souligne l'importance de l'antagonisme entre ces 2 composantes (fig. 5, $\mathrm{r}=-0,85$ ).

Il s'ensuit que ce sont les génotypes triticale I.N.R.A. qui assurent les meilleures productions moyennes (environ $300 \mathrm{~kg}$ de protéines/ha), suivis des populations de seigles (environ $240 \mathrm{~kg}$ ). Les calculs de régressions $\mathrm{y}_{\mathrm{ij}} / \mathrm{y} \cdot \mathrm{j}$ font apparaître, comme pour les autres caractères, une relative indépendance des seigles par rapport aux productions moyennes des différents milieux : les coefficients de corrélation sont relativement faibles $(r=0,8$, à la limite de signification au seuil 0,05 ) et les coefficients de régression très inférieurs à l'unité (de l'ordre de 0,55 à 0,6 ). Pour les 3 autres groupes, les coefficients de corrélation sont très hautement significatifs et les coefficients de régression

\section{TABLEAU 5}

Analyses de variance pour le poids de 1000 grains (PMG), la teneur en protéines (TP), la production de protéines par hectare (PP) suivant MANDEL (1971).

Analysis of variance: 1000 kernel weight (PMG), protein content (TP), protein production per hectare (PP), according to MANDEL (1971).

\begin{tabular}{|c|c|c|c|c|c|c|c|c|c|c|}
\hline \multirow{2}{*}{$\begin{array}{c}\text { Origine } \\
\text { de la variation }\end{array}$} & \multirow[b]{2}{*}{ DDL } & \multicolumn{3}{|c|}{ PMG (g) } & \multicolumn{3}{|c|}{ TP (\% de la M.S.) } & \multicolumn{3}{|c|}{ PP (kg/ha) } \\
\hline & & SCE & $\mathrm{CM}$ & $\mathrm{F}$ & SCE & $\mathrm{CM}$ & F & SCE & $\mathrm{CM}$ & $\mathrm{F}$ \\
\hline Totale & 104 & 7802,0 & 75,02 & - & 791,8 & 7,61 & - & 2487017 & 23914 & - \\
\hline Licux & 4 & 1502,4 & 375,6 & $29,7 * *$ & 124,1 & 31,04 & $36,43 * *$ & 1249533 & 312383 & $4586 * *$ \\
\hline Genotypes & 20 & 5289,2 & 264,5 & $20,9 * *$ & 599,5 & 29,98 & $35,18 * *$ & 692540 & 34627 & $5,088 * *$ \\
\hline Interaction & 80 & 1010,5 & 12,6 & - & 68,2 & 0,85 & - & 544943 & 6811 & - \\
\hline & 23 & 670,5 & 29,15 & U $66,35 * *(1)$ & 38,7 & 1,68 & $U=56,7^{* *(1)}$ & 475274 & 20664 & $\mathrm{U}=87,2 * *(1)$ \\
\hline Résiduelle & 57 & 340 & 5,96 & - & 29,5 & 0,52 & - & 69669 & 1222 & - \\
\hline
\end{tabular}

(1) Test U de Johnson \& Graybill (1972) exprimé en p. 100. 


\section{TABLEAU 6}

Valeurs moyennes mesurées sur les différents génotypes pour les caractères: poids de 1000 grains (PMG), teneurs en protéines (TP) et production de protéines par hectare (PP).

Mean values observed in the different genotypes for the characters : 1000 -kernel weight (PMG), protein content (TP) and protein production per hectare (PP).

\begin{tabular}{|c|c|c|c|}
\hline & PGM (g) & TP (\% de M.S.) & PP (kg/ha) \\
\hline $\begin{array}{l}\text { Blés Barbela } \\
\text { Autonomia } \\
\text { Siete Cerros }\end{array}$ & $\begin{array}{l}51,85 \\
36,85 \\
31,35\end{array}$ & $\begin{array}{r}9,3 \\
10,0 \\
9,6\end{array}$ & $\begin{array}{r}104 \\
89 \\
104\end{array}$ \\
\hline $\begin{array}{c}\text { Seigles Vila Pouca } \\
\text { Lordelo } \\
\text { Montalegre } \\
\text { Gimonde }\end{array}$ & $\begin{array}{l}29,6 \\
31,8 \\
30,0 \\
30,6\end{array}$ & $\begin{array}{l}8,8 \\
9,8 \\
9,1 \\
9,5\end{array}$ & $\begin{array}{l}245 \\
231 \\
220 \\
232\end{array}$ \\
\hline $\begin{array}{c}\text { Triticale INRA } \\
532-43 \\
533-A-10 \\
532-6 \\
21 \\
15 \\
9 \\
4\end{array}$ & $\begin{array}{l}47,5 \\
52,4 \\
47,0 \\
46,8 \\
44,2 \\
45,4 \\
46,4\end{array}$ & $\begin{array}{l}8,4 \\
9,5 \\
9,0 \\
8,5 \\
8,3 \\
8,7 \\
7,8\end{array}$ & $\begin{array}{l}294 \\
262 \\
280 \\
304 \\
309 \\
292 \\
327\end{array}$ \\
\hline $\begin{array}{l}\text { Triticale CIMMYT } \\
\text { Armadillo } \\
\text { Inia-ArmS (307) } \\
\text { Maya II-ArmS (309) } \\
\text { Cinnamon (306) } \\
\text { Maya II-ArmS (300) } \\
\text { Maya II-ArmS (308) } \\
\text { Beagle (17) }\end{array}$ & $\begin{array}{l}39,0 \\
38,3 \\
41,3 \\
37,5 \\
35,6 \\
42,9 \\
47,0\end{array}$ & $\begin{array}{l}15,2 \\
13,5 \\
13,5 \\
15,6 \\
13,8 \\
12,6 \\
12,4\end{array}$ & $\begin{array}{r}101 \\
96 \\
119 \\
92 \\
114 \\
207 \\
225\end{array}$ \\
\hline PPDS & 1,13 & 0,29 & 26,2 \\
\hline
\end{tabular}

TABLEAU 7

Valeurs moyennes mesurées dans les différents lieux pour les caractères: poids de 1000 grains $(P M G)$, teneur en protéines (TP) et production de protéines par hectare (PP).

Mean values observed in the different locations for the following characters : 1000 kernel-weight $(P M G)$, protein content $(T P)$ and protein production per hectare $(P P)$.

\begin{tabular}{|c|c|c|c|c|c|}
\hline & \multicolumn{2}{|c|}{ Vila Real } & \multirow{2}{*}{$\begin{array}{c}\text { Carlão } \\
\text { C }\end{array}$} & \multirow{2}{*}{$\begin{array}{c}\text { Montalegre } \\
\mathbf{M}\end{array}$} & \multirow{2}{*}{$\begin{array}{c}\text { Bragança } \\
\text { B }\end{array}$} \\
\hline & TT2 & TT3 & & & \\
\hline PMG (g) & 46,4 & 39,0 & 43,8 & 37,3 & 36,8 \\
\hline TP (\% MS) & 12,7 & 9,9 & 10,7 & 9,9 & 9,9 \\
\hline PP (kg) & 383 & 117 & 280 & 157 & 95 \\
\hline
\end{tabular}

Figure 3

Droites de régression du rendement au poids de 1000 grains observées pour les différents groupes de génotypes.

Regression of yield to the 1000-kernel weight observed for the different groups of genotypes.

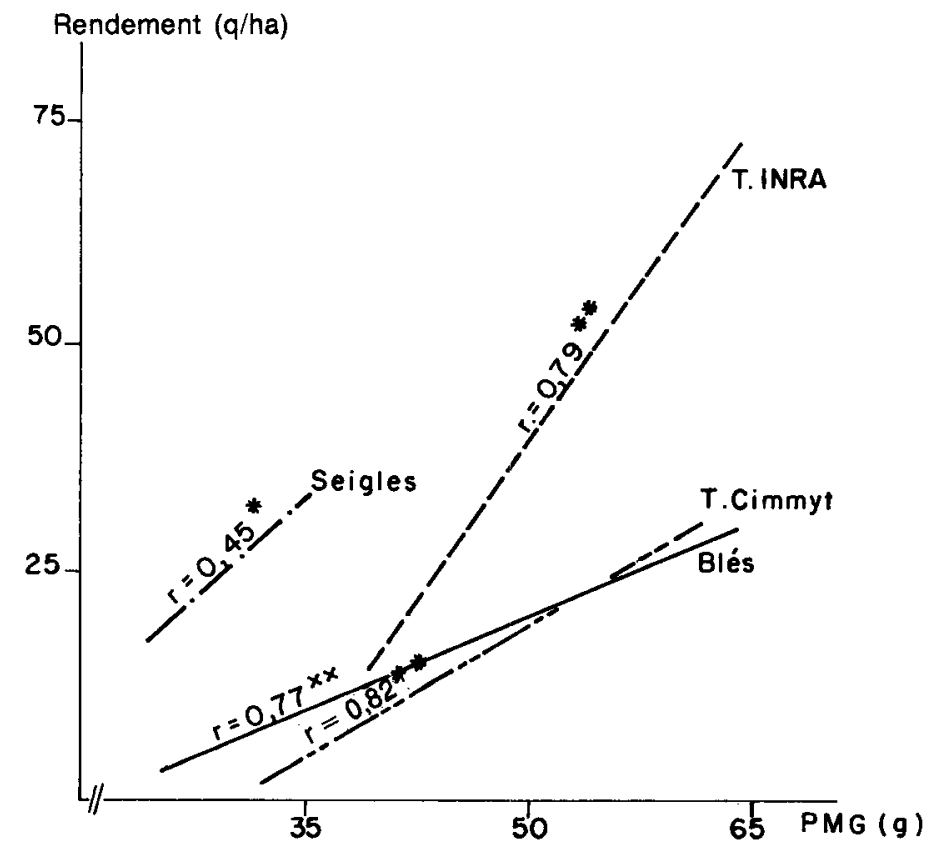



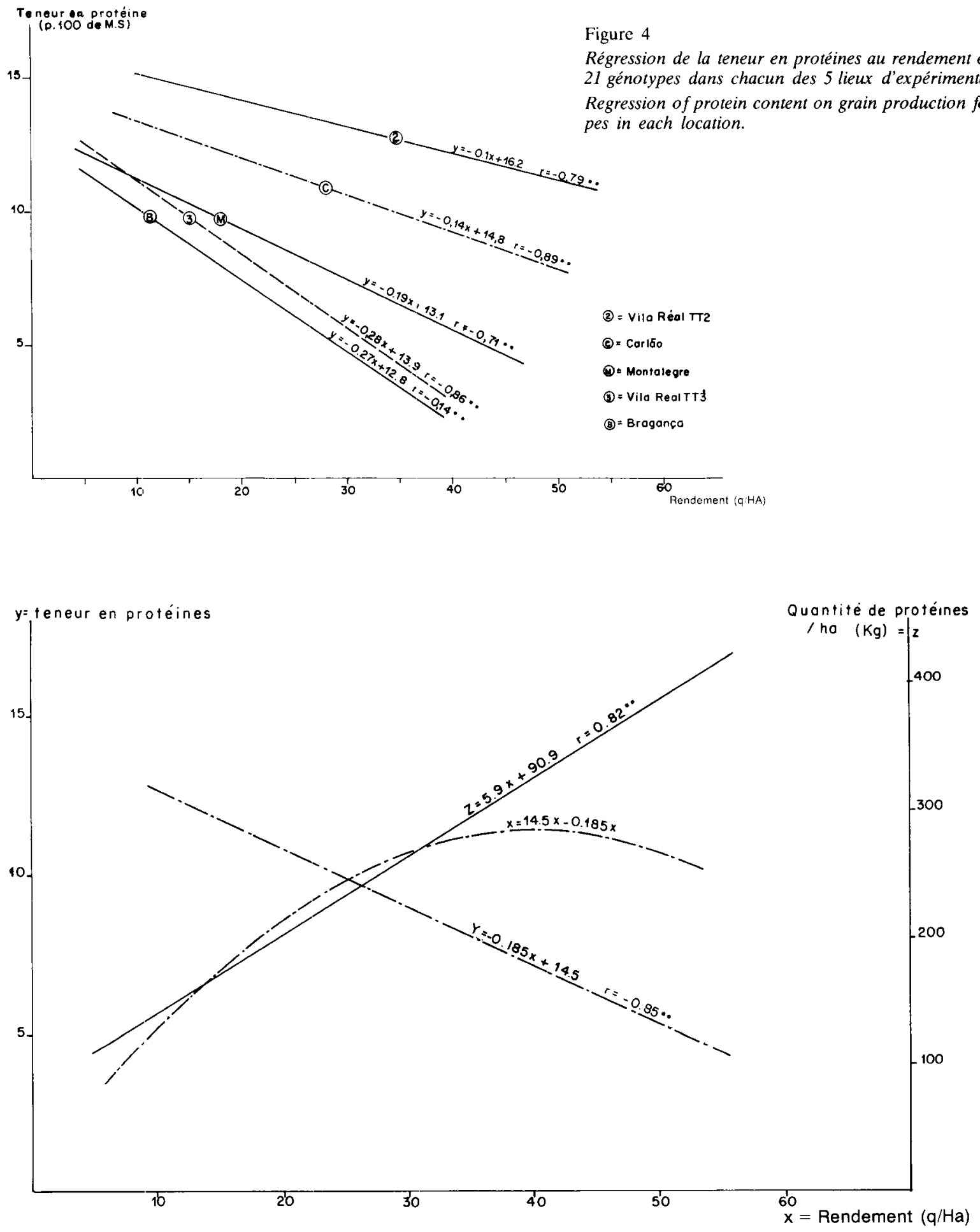

Figure 5

Régression de la teneur en protéines y (-. - .) et de la production de protéines z (-) au rendement en grain sur les valeurs moyennes de 21 génotypes (premier et second degrés).

varient de 0,57 (pour les blés) à 1,7 (pour les triticales I.N.R.A.) : ces groupes sont plus sensibles à l'influence du milieu.

\section{DISCUSSION ET CONCLUSION}

Aucun des génotypes testés ne présente régulièrement les meilleures performances dans tous les milieux. Cependant, 2 groupes de génotypes présentent les performances les plus
Regression of protein content $y(-.-$.$) and protein production z$ $(-)$ on grain production for the mean values of the 21 genotypes.

satisfaisantes dans 2 types de milieux

- les populations locales de seigle dans les «milieux pauvres de montagne" mais dont les rendements plafonnent entre 30 et $40 \mathrm{q} /$ ha et s'effondrent quand le milieu devient trop riche ;

- les lignées ae triticale I.N.R.A., dont la production est médiocre dans les milieux pauvres mais qui exploitent beaucoup mieux que les populations de seigle les terres riches des vallées (jusqu'à 70 q/ha). 


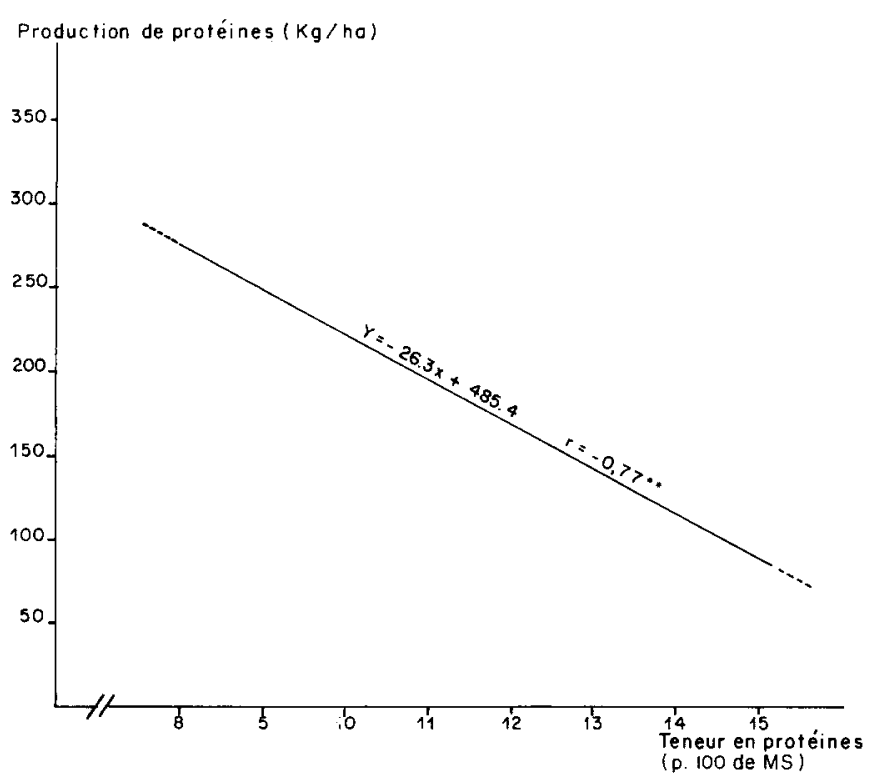

Figure 6

Régression de la production de protéines à la teneur en protéines du grain calculée sur les valeurs moyennes des 21 génotypes.

Regression of protein production on protein content for the mean values of the 21 genotypes.

Nous retrouvons là les 2 groupes de génotypes définis précédemment: l'un à production élevée et à forte dépendance du milieu, l'autre à production moyenne et à faible dépendance du milieu. Un travail de sélection devrait évidemment s'attacher à obtenir des « recombinants favorables $»$ entre les 2 types.

Les génotypes des 2 autres groupes ne présentent de performances satisfaisantes dans aucun des milieux, à l'exception du blé «Barbela » qui se comporte à certains égards comme un seigle, bien qu'étant très différent quant à certaines composantes du rendement (poids de 1000 grains notamment) ; ceci montre qu'un blé peut être aussi « rustique » qu'un seigle. Les propriétés adaptatives peuvent donc être acquises indépendamment du système. de biologie florale.

Il convient de noter que la composante du rendement «poids de 1000 grains » est à peu près indépendante du milieu pour « Barbela » aussi bien que pour les seigles. Une telle absence de variation est évidemment pénalisante en milieux riches, mais représente une sécurité en milieux $\mathrm{x}$ pauvres. Il serait inréressant de voir si cette particularité se vérifie au niveau des autres composantes du rendement aussi bien qu'au niveau des compensations entre composantes.

Les résultats que nous avons observés quant aux productions de protéines peuvent sembler d'interprétation difficile, notamment le fait qu'elles soient liées positivement, et à un tel degré, à la production de matière sèche dans le grain, et négativement aux teneurs en protéines. En fait, si nous considérons les relations existant entre la quantité y de protéines produite en un lieu par un génotype, la quantité $x$ de matière sèche (grain) fournie par le génotype, enfin la relation existant entre la teneur en protéines ( $t$ ) dans le grain et la quantité $x$, relation du type :

$$
t=-a x+b
$$

on a alors :

$$
y=-a x^{2}+b x
$$

$c^{\prime}$ est-à-dire une courbe du $2^{c}$ degré passant par un optimal.

Si l'on retient pour valeurs approchées de $a$ et $b$ celles correspondant à la régression sur les valeurs moyennes : teneur en protéines/quantité de grain $(a=0,185$; $b=14,5$ ), l'optimal est atteint dans les conditions de cette expérimentation pour des valeurs de $\mathrm{x}$ de l'ordre de 60 à $70 \mathrm{q} / \mathrm{ha}$; les valeurs de y seront au plus égales à $500 \mathrm{~kg}$. Tous les résultats expérimentaux se situent dans la partie croissante de la courbe, ce qui explique les types de réponse obtenus.

Bien que ce paramètre n'ait pas été pris en compte dans l'analyse, les 2 groupes présentant les performances agronomiques les plus satisfaisantes ont des hauteurs de chaume élevées. Au contraire, tous les génotypes de taille relativement courte semblent impuissants à exploiter la gamme de milieux proposée. De plus, il convient de remarquer que la production de paille est relativement moins variable que la production de grain : écart de 1 à 2 dans le $1^{\text {er }}$ cas, de 1 à 6 dans le $2^{\mathrm{c}}$, au niveau des moyennes variétales. Autrement dit, une diminution des possibilités de production de matière sèche totale retentit surtout sur l'aptitude au stockage dans le grain. Au niveau de l'« Harvest Index», ceci se traduit par des valeurs variétales d'autant plus basses que la production totale de matière sèche assurée par la plante est faible; cette production est statistiquement d'autant plus faible que la plante est courte. La régularité de production en grain sera donc mieux assurée avec des génotypes « hauts " qu'avec des génotypes «courts ", pour lesquels on a pensé, initialement, que les transferts et le stockage de matière sèche au niveau de l'épi se feraient de façon plus efficace. Il est possible que cette hypothèse se vérifie dans les milieux très favorables et techniquement évolués. Elle est infirmée pour la gamme de milieu entrant dans notre expérimentation.

La précocité a également pu jouer un rôle. Cependant, le fait que les seigles régionaux et les triticales CIMMYT ont la même précocité de floraison et de maturation tend à infirmer ce seul aspect, d'autant que la sécheresse estivale prononcée aurait dû désavantager les variétés tardives (matériel I.N.R.A.).

Cette expérimentation souligne enfin l'intérêt que peut présenter un matériel traditionnellement cultivé dans une région quant à sa valeur d'adaptation moyenne à cette région. Il est évident qu'il est nécessaire d'exploiter au mieux la richesse génétique qu'il représente, pour la zone considérée évidemment, mais aussi sur un plan plus général. L'élimination massive et sans étude préalable de ce type de matériel constituerait manifestement une faute agronomique et scientifique. Le programme qui a débuté à Vila Real consistera donc à utiliser au mieux les potentialités mises en évidence au niveau des seigles locaux et de «Barbela » dans un programme d'amélioration du triticale.

Reçu le 16 février 1982. Accepté le 23 mars 1983. 


\section{RÉFÉRENCES BIBLIOGRAPHIQUES}

Anonyme, 1976. O Instituto Politecnico de Vila Real. Area de Influencia Objectives. Necessidades. IPVR Vila Real, $20 \mathrm{p}$.

Bernard M., Branlard G., Farouk-el-Tallawi M., Laroche G., Bourdet A., Feillet P., 1979. Caractéristiques quantitatives et qualitatives des protéines de Triticale : études de lignées sélectionnées en France. Seed Protein Improvement in Cereals and Grain Legumes. Proc. Int. Symp., Neuherberg, 4-8 September 1978, Vol. II, p. 237-259, IAEA Vienne (1979), sti/pub/496.

Brian C., 1978. Interprétation statistique des essais « deux voies" : décomposition factorielle des résidus et étude de la structure des interactions. Ann. Amélior. Plantes, 28 (4), 395-410.

Cauderon Y., Bernard M., 1980. Yield improvement from $(8 \mathrm{x} \times 6 \mathrm{x})$ crosses, and genic and cytoplasmic diversification in Triticale. Hod. Rosl. Aklim. Nesien, 24 (4), 329-338.

Guedes Pinto H., Bernard M., 1980. Analysis of similarities between some cultivars of wheat, rye and Triticale for morphological and yield components and their genotype-environment interac- tion in two different soil fertility conditions. Hod. Rosl. Aklim. Nesien, 24, 5, 631-644.

Johnson D. E., Graybill A., 1972. An analysis of a two-ways model with interaction and no replication. J. Am. Statist. Assoc., 67, 340, 862-868.

Jestin L., 1974. Les variations de la teneur en protéines brutes chez l'orge selon la variété et le milieu : étude préliminaire à partir d'essais culturaux. Ann. Amélior. Plantes, 24 (4), 377-388.

Lefort P. L., 1977. L'interaction entre facteurs dans l'analyse de variance. Application à l'interaction génotype $\times$ milieu. $T D R$, $\mathrm{n}^{\circ} 6$, p. 444-453.

Mandel J., 1971. A new analysis of variance model for non additive data. Technometrics, 13, 1-18.

Wright A. J., 1976. The significance for breeding of linear regression analysis of genotype-environment interactions. Heredity, 37 (1), 89-93. 